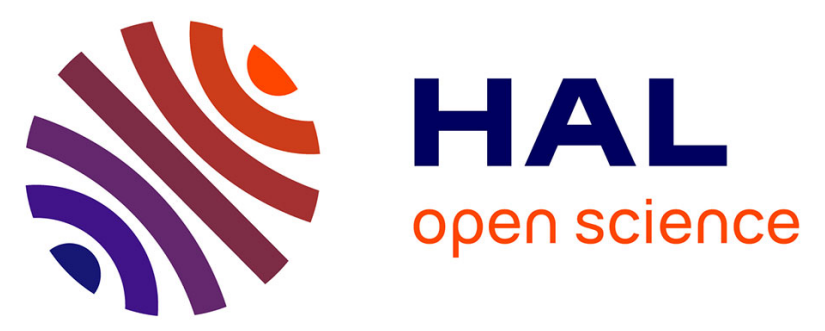

\title{
Simultaneous Air Fraction and Low-Pressure EGR Mass Flow Rate Estimation for Diesel Engines
}

Felipe Castillo Buenaventura, Emmanuel Witrant, Vincent Talon, Luc Dugard

\section{To cite this version:}

Felipe Castillo Buenaventura, Emmanuel Witrant, Vincent Talon, Luc Dugard. Simultaneous Air Fraction and Low-Pressure EGR Mass Flow Rate Estimation for Diesel Engines. IFAC Joint conference SSSC - 5th Symposium on System Structure and Control, Feb 2013, Grenoble, France. hal-00752315

\section{HAL Id: hal-00752315 \\ https://hal.science/hal-00752315}

Submitted on 15 Nov 2012

HAL is a multi-disciplinary open access archive for the deposit and dissemination of scientific research documents, whether they are published or not. The documents may come from teaching and research institutions in France or abroad, or from public or private research centers.
L'archive ouverte pluridisciplinaire $\mathbf{H A L}$, est destinée au dépôt et à la diffusion de documents scientifiques de niveau recherche, publiés ou non, émanant des établissements d'enseignement et de recherche français ou étrangers, des laboratoires publics ou privés. 


\title{
Simultaneous Air Fraction and Low-Pressure EGR Mass Flow Rate Estimation for Diesel Engines
}

\author{
F. Castillo* E. Witrant ${ }^{* *}$ V. Talon* L. Dugard ${ }^{* *}$ \\ * Renault SAS, 1 Allee Cornuel, 91510 Lardy, France (e-mail: \\ felipe.castillo,vincent.talon@lrenault.com) \\ ** UJF-Grenoble1/CNRS, Grenoble Image Parole Signal Automatique \\ (GIPSA-Lab), UMR5216, B.P. 46, 38402 St Martin d'Heres, France \\ (e-mail: emmanuel.witrant,luc.dugard@gipsa-lab.fr)
}

\begin{abstract}
This paper describes a low-pressure exhaust gas recirculation (LP-EGR) mass flow rate estimation method and a robust air mass fraction observer for a Diesel engine with dualloop EGR system. Both observers operate simultaneously eliminating the need for pressure measurement upstream the LP-EGR valve. A sliding mode observer is designed to estimate the LP-EGR mass flow rate using the standard sensors available in commercial Diesel engines. A robust linear parameter varying Kalman filter is designed for the air mass fraction estimation. The convergence and robustness of the observers are ensured by means of Lyapunov stability and a linear matrix inequality (LMI) framework for the sliding mode observer and robust Kalman filter, respectively. The observers are validated with a Motor Vehicle Emission Group (NMVEG) cycle using an engine model validated on an experimental benchmark as a reference.
\end{abstract}

\section{INTRODUCTION}

In the recent years, Diesel engine emissions regulations have become stricter and achieving simultaneously the emissions legislations and the demanded engine drivability has become a very challenging issue. Although significant improvements have been made over the past years, there are still many challenges to address in order to meet the future emissions regulations. The introduction of sophisticated alternative combustion modes such as homogeneous charge compression ignition (HCCI) and low temperature combustion (LTC) a offer a great potential to reduce the engine emissions levels (see Akihama et al. [2001] Alriksson and Denbrantt [2006] Ryan and Matheaus [2003]). However, these new modes require different fueling strategies and in-cylinder conditions, thus creating the need for more complex, reliable and precise control systems and technologies.

Dual-loop exhaust gas recirculation (EGR) with both high and low-pressure recirculations, is one of the new strategies proposed to achieve the appropriate conditions to implement multiple combustion modes (see Hribernik [2002]). However, ensuring the adequate in-cylinder conditions is still a very difficult task, as the introduction of the EGR implies to solve many control challenges due to the lack of EGR flow rates and mass fraction measurements. An efficient control of the in-cylinder combustion and engineout emissions not only involves the total in-cylinder EGR amount, but also the ratio between the high-pressure EGR (HP-EGR) and the low-pressure EGR (LP-EGR). Indeed, this ratio is crucial as the gas temperatures and compositions are significantly affected. The HP-EGR gas is helpful to stabilize the combustion at low load since its temperature is high. The LP-EGR reduces the engineout NOx emission without excessive smoke as it is filtered by the particle filter. Controlling the air fractions in the intake manifold is an efficient approach to control the incylinder EGR amount (see Ammann et al. [2003] Chauvin et al. [2006]). For engines with dual EGR systems, the air fraction upstream of the compressor provides the LP-EGR rate and the air fraction in the intake manifold provides the total EGR rate. Therefore, if the air fractions in each section are controlled, then the HP and LP-EGR can also be controlled efficiently.

The EGR mass flow rates can be estimated by measuring the upstream and downstream pressures and the upstream temperature of the EGR valves along with the orifice equation and an empirical function describing the valve's effective area as a function of its position. Nevertheless, this EGR air mass rate estimation is an expensive solution as sensors have to be installed. Moreover, in the case of LP-EGR. This measurement is inaccurate, because of the small pressure drop across the LP-EGR valve. This motivates the search for alternative strategies to determine the EGR mass flow rates.

Controlling the air mass fraction is a difficult task, because its direct measurement is not available on the production engines and the dynamics of the admission air-path can be highly complex. Therefore, several air fraction/EGR rate estimation methods have been proposed in the literature. In Chauvin et al. [2006] Kolmanovski et al. [2000], estimators of burned gas fraction in the intake manifold have been designed for single loop EGR. In Wang [2008], a method for estimating the air fraction in each section of 
the engine has been proposed for dual-loop EGR systems. However, estimating the air fraction simultaneously with LP-EGR mass flow rate has been less explored. In this paper, new methods to estimate the LP-EGR mass flow rate and to robustly observe the air fraction are proposed, based on a 0D modeling approach. The observers are derived to work simultaneously, reducing the need for measuring the pressure upstream of the LP-EGR valve to estimate the air fraction in the intake manifold.

This paper is organized as follows: the intake manifold pressure and air fraction dynamic models are presented in Section 2. In Section 3, the engine general control architecture is described. In Section 4, the sliding mode framework is used to estimate the LP-EGR mass flow rate from the pressure measured in the intake manifold. Its convergence is proved using Lyapunov stability. In Section 5, an air fraction observer is designed using a robust LPV Kalman filter along with a synthesis based on linear matrix inequalities (LMI), which ensure the convergence and robustness of the estimate. Finally, the performance of the observers working simultaneously is evaluated using a validated engine model as a reference.

\section{ENGINE AIR PATH MODELING}

The air path model considered in this section is based on a modern light-duty four-cylinder Diesel 1.6 liter engine. Its scheme is depicted in Figure 1.

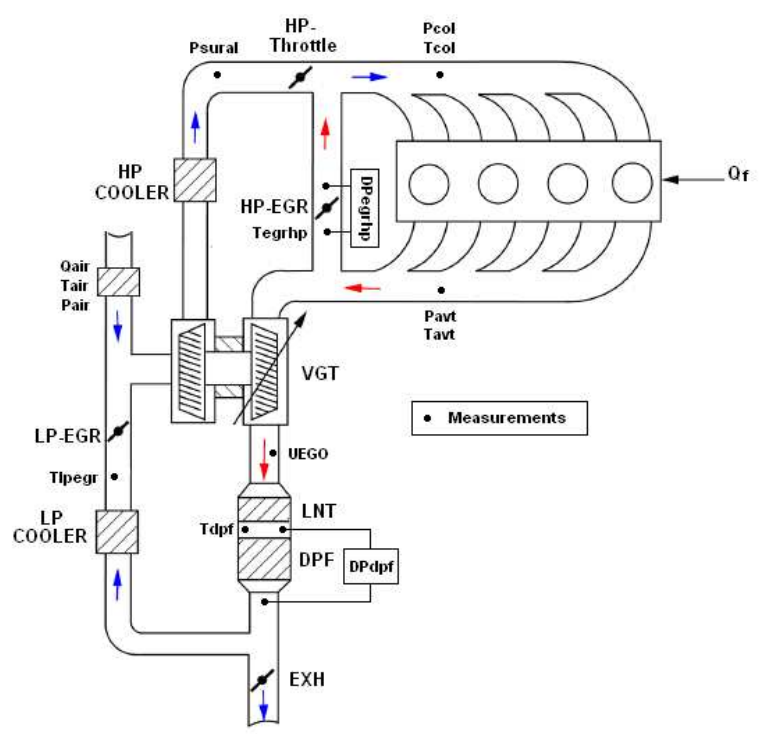

Fig. 1. Schematic description of the Dual-Loop EGR with VGT

The engine is equipped with a variable geometry turbocharger (VGT), a dual-loop EGR system (high-pressure throttle, as well as low-pressure throttle) and exhausttreatment systems such as a Diesel particle filter and a lean NOx trap. A high-pressure throttle (denoted as HP-throttle) increases the HP-EGR rate at light load. A universal exhaust gas oxygen (UEGO) sensor is installed downstream the VGT in order to avoid high-pressure at the UEGO sensor. For Diesel engines, the combustion is usually lean, which means that there is excessive air (in comparison with the stoichiometric amount) in the cylinder mixture. Therefore, the exhaust gas contains unburned air and it could be recirculated back into the intake manifold through the EGR valves. The air mass fraction is very important for combustion performance and emissions reduction, specially for alternative combustion modes. The HP-EGR is used during the beginning of a cycle in order to obtain combustions with more elevated temperature, with the purpose of heating up the exhaust post-treatment systems as quickly as possible. The LP-EGR is then used instead of the HP-EGR because it is cooler, thus allowing to introduce more mass and EGR into the cylinder.

In order to build a reduced model of the engine intake manifold, the following assumptions are made:

A-1 the dynamics of the temperature is much slower than the pressure dynamics;

A-2 the volumes associated with the exhaust gas recirculation are considered small;

A-3 the HP-throttle is completely open during LP-EGR operation.

Similarly to the models proposed in Wang [2008] and Chauvin et al. [2008], the dynamics of the intake manifold can be approximated by:

$$
\begin{aligned}
& \dot{p}_{c o l}=\frac{R T_{c o l}}{V_{e q v}}\left(Q_{a i r}+Q_{e g r l}+Q_{e g r h}-Q_{m o t}\right) \\
& \begin{array}{c}
\dot{F}_{a v t}=\frac{R T_{a v t}}{p_{a v t} V_{a v t}}\left(\left(Q_{a i r}+Q_{e g r l}+Q_{e g r h}\right) F_{c o l}-\left(Q_{a i r}+\right.\right. \\
\left.\left.Q_{e g r l}+Q_{e g r h}+Q_{f}\right) F_{a v t}-P C O Q_{f}\right)
\end{array} \\
& \dot{F}_{a v c}=\frac{R T_{a i r}}{p_{a i r} V_{a v c}}\left(\left(F_{a v t}-1\right) Q_{e g r l}+\left(1-F_{a v c}\right)\right. \\
& \left.\left(Q_{\text {air }}+Q_{\text {egrl }}\right)\right) \\
& \dot{F}_{\text {sural }}=\frac{R T_{\text {sural }}}{p_{\text {sural }} V_{\text {sural }}}\left(Q_{\text {air }}+Q_{\text {egrl }}\right)\left(F_{\text {avc }}-F_{\text {sural }}\right) \\
& \dot{F}_{\text {col }}=\frac{R T_{\text {col }}}{p_{\text {col }} V_{\text {col }}}\left(\left(Q_{\text {air }}+Q_{\text {egrl }}\right)\left(F_{\text {sural }}-F_{c o l}\right)+\right. \\
& \left.Q_{\text {egrh }}\left(F_{\text {avt }}-F_{\text {col }}\right)\right)
\end{aligned}
$$

where $p, T, F$ and $V$ stand for pressure, temperature, air fraction and volume, respectively, and the indexes col, avt, avc, sural and air correspond to the intake manifold, exhaust manifold, upstream the compressor, between the compressor and the HP-throttle and atmospheric conditions, respectively. For example, $F_{\text {sural }}$ is the air mass fraction between the compressor discharge and the HPthrottle. $R$ is the specific gas constant, $P C O$ is the stoichiometric air to fuel ratio and $Q_{f}, Q_{a i r}, Q_{m o t}, Q_{e g r h}$ and $Q_{\text {egrl }}$ are the fuel, the fresh air, the engine admission, the high-pressure and low-pressure EGR mass flow rates, respectively. $V_{\text {eqv }}$ is an equivalent volume for the 0D pressure dynamics defined as $V_{e q v}=V_{a p c}+V_{a p e}+V_{h e}+V_{c o l}$ where $V_{a p c}$ is the volume between the compressor and the HPcooler, $V_{h e}$ is the HP-cooler volume and $V_{a p e}$ is the volume 
between the HP-cooler and the HP-throttle. According to the schematic presented in Figure 1, the only measured states are $p_{\text {col }}$ and $F_{\text {avt }}$ (UEGO sensor). Note that $p_{a v t}$, $T_{a v t}, T_{c o l}$ and $Q_{a i r}$ are measured directly in the engine. The value of $Q_{m o t}$ results from an empirical function or data-map depending usually on $p_{c o l}, T_{c o l}$ and the engine speed $N_{m o t}$

$Q_{\text {egrh }}$ and $Q_{\text {egrl }}$ still have to be taken into account in order to complete the information to solve system (1)(5). These variables have a large degree of uncertainty due to the inaccurate measurement of the differential pressure through the EGR valves and the uncertainties on the valve's effective empirical function. It would be desirable to achieve an estimation of both mass flows rates from system (1)-(5), but according to the measurements available on the engine described in Figure 1, $Q_{\text {egrh }}$ and $Q_{\text {egrl }}$ cannot be estimated at the same time. As $p_{c o l}$ is measured, it is possible to solve (1) by searching the right value for the sum $Q_{\text {egrh }}+Q_{\text {egrl }}$. However, it is clear that $Q_{\text {egrh }}$ and $Q_{\text {egrl }}$ cannot be distinguished. The differential pressure across the low-pressure EGR valve is much smaller than the one exhibited by the HP-EGR valve, making this measurement more complex and more inaccurate. That is why an estimation of the low-pressure EGR mass flow rate is considered in this work.

The dynamics of the intake manifold pressure is much faster than the dynamics of the air mass fraction and the air fraction does not influence the pressure dynamics, as can be seen in (1)-(5). These properties allow considering the air fraction and LP-EGR mass flow rate estimation as two separate problems.

\section{AIR FRACTION GENERAL CONTROL ARCHITECTURE}

Figure 2 shows the air path control architecture considered in this work. The first part, from left to right, corresponds to the engine mapping resulting from a complex calibration phase, not detailed in this work. The intake manifold pressure, air fraction and EGR proportion $\left(p_{\text {colsp }}, F_{\text {colsp }}\right.$ and $\% E G R_{s p}$, respectively) are mapped according to an indicated mean effective pressure (IMEP) set-point imposed by the driver and the measured engine speed $N_{m o t}$. $\% E G R_{s p}$ determines the EGR proportion that must be applied.

The air fraction controller is a state feedback based on the air fraction estimation at each section of the airpath. The inputs associated with the air fraction observer are the measurements taken directly or indirectly in the engine (presented in Figure 2 as $\Psi$ ). The outputs are the estimated air fractions at each section of the air path. The air fraction controller outputs are the HP and LP EGR mass flows rates which are then transformed into valve position. The boost pressure controller is not considered in this work.

\section{LOW PRESSURE EGR FLOW RATE OBSERVER DESIGN}

In this section, an observer is designed to obtain an estimation of $Q_{e g r l}$, denoted as $\hat{Q}_{e g r l}$. This estimation can not be directly done because $Q_{\text {egrl }}$ is not a state space variable but a system's input. Consider the state extension $\dot{Q}_{\text {egrl }}=0$ and the following observer architecture to include the variations of $\hat{Q}_{\text {egrl }}$ :

$$
\begin{gathered}
\dot{\hat{p}}_{c o l}=\frac{R T_{c o l}}{V_{e q v}}\left(Q_{a i r}+\hat{Q}_{e g r l}+Q_{e g r h}-Q_{m o t}\right)+u_{1} \\
\dot{\hat{Q}}_{e g r l}=u_{2}
\end{gathered}
$$

The estimation problem is to find $u_{1}$ and $u_{2}$ such that the convergence to zero of the estimation errors $p_{\text {col }}-\hat{p}_{c o l}$ and $Q_{\text {egrl }}-\hat{Q}_{\text {egrl }}$ is guaranteed. Different state estimation strategies may be used. However, due to the fact that system (6) - (7) is already in an additive triangular form, a sliding mode observer (suitable for this kind of systems) is chosen because of its inherent robustness and its implementation simplicity (see Perruquetti and Barbot [2002]).

Define the first sliding surface with integral action as:

$$
S_{1}=k_{1}\left(\hat{p}_{c o l}-p_{c o l}\right)+k_{2} \int\left(\hat{p}_{c o l}-p_{c o l}\right) d t
$$

where $k_{1}$ and $k_{2}$ are some constant parameters. Consider the following Lyapunov function candidate:

$$
V(t)=\frac{1}{2} S_{1}^{2}(t)
$$

Taking the derivative of $V$ along the trajectories of (6) gives:

$$
\begin{aligned}
\dot{V}=S_{1} & \left(k_{1}\left(\frac{R T_{\text {col }}}{V_{\text {eqv }}}\left(\hat{Q}_{\text {egrl }}-Q_{\text {egrl }}\right)+u_{1}\right)\right. \\
+ & S_{1} k_{2}\left(\hat{p}_{c o l}-p_{c o l}\right)
\end{aligned}
$$

Define $u_{1}$ as:

$$
u_{1}=h_{1} \lambda_{1} \operatorname{sign}\left(S_{1}\right)+h_{2}\left(\hat{p}_{c o l}-p_{c o l}\right)
$$

where $h_{1}$ and $h_{2}$ are time-varying variables precised later and $\lambda_{1}$ is a constant. This leads to:

$$
\begin{gathered}
\dot{V}=S_{1}\left(k_{1} \frac{R T_{c o l}}{V_{e q v}}\left(\hat{Q}_{e g r l}-Q_{e g r l}\right)+k_{1} h_{1} \lambda_{1} \operatorname{sign}\left(S_{1}\right)\right. \\
\left.+k_{1} h_{2}\left(\hat{p}_{c o l}-p_{c o l}\right)\right)+S_{1} k_{2}\left(\hat{p}_{c o l}-p_{c o l}\right)
\end{gathered}
$$

Choosing $h_{1}$ and $h_{2}$ as follows:

$$
h_{1}=\frac{R T_{c o l}}{V_{e q v}}, \quad h_{2}=-\frac{k_{2}}{k_{1}}
$$




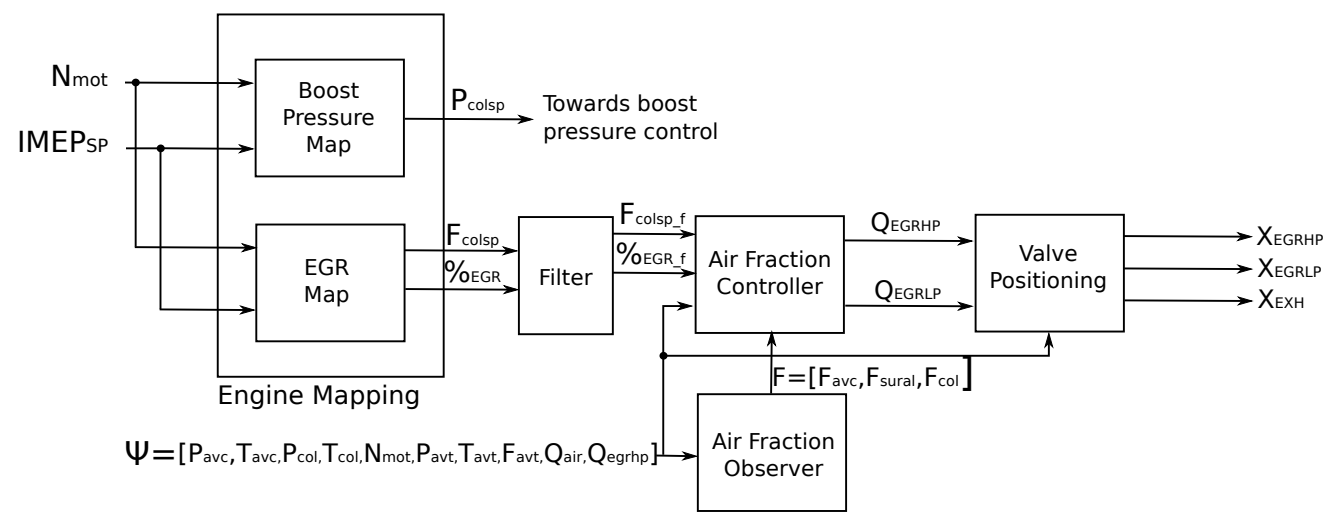

Fig. 2. Air Fraction Control Architecture

gives:

$$
\dot{V}=S_{1} k_{1} \frac{R T_{\text {col }}}{V_{\text {eqv }}}\left(\hat{Q}_{e g r l}-Q_{e g r l}+\lambda_{1} \operatorname{sign}\left(S_{1}\right)\right)
$$

In order to ensure the asymptotic stability, $\lambda_{1}$ and $k_{1}$ are chosen as:

$$
\left|\hat{Q}_{e g r l}-Q_{e g r l}\right|<\left|\lambda_{1}\right|, \quad \lambda_{1}<0, \quad k_{1}>0
$$

It can be easily shown that when the estimation error $e=\hat{p}_{c o l}-p_{c o l}$ is at steady state $(\dot{e}=0)$, the following expression is obtained:

$$
Q_{e g r l}=\hat{Q}_{e g r l}+\lambda_{1} \operatorname{sign}\left(S_{1}\right)-\frac{k_{2}}{k_{1}} \frac{V_{e q v}}{R T_{c o l}}\left(\hat{p}_{c o l}-p_{c o l}\right)
$$

(16) permits to define a second sliding surface as follows:

$$
S_{2}=k_{3}\left(\hat{Q}_{e g r l}-Q_{e g r l}\right)+k_{4} \int\left(\hat{Q}_{e g r l}-Q_{e g r l}\right) d t
$$

where $k_{3}$ and $k_{4}$ are constants. Using the same Lyapunov function candidate (9) for the sliding surface $S_{2}$, denoted as $V_{2}$, the following is obtained after taking into account that $\dot{Q}_{\text {egrl }}=0$ (defined for the state insertion):

$$
\dot{V}_{2}=S_{2}\left(k_{3} u_{2}+k_{4}\left(\hat{Q}_{e g r l}-Q_{e g r l}\right)\right)
$$

Define $u_{2}$ as:

$$
u_{2}=\lambda_{2} \operatorname{sign}\left(S_{2}\right)+h_{3}\left(\hat{Q}_{e g r l}-Q_{e g r l}\right)
$$

where $\lambda_{2}$ and $h_{3}$ are constant tuning parameters. Then, (18) can be written as:

$$
\begin{aligned}
& \dot{V}_{2}=S_{2}\left(k_{3}\left(\lambda_{2} \operatorname{sign}\left(S_{2}\right)+h_{3}\left(\hat{Q}_{e g r l}-Q_{e g r l}\right)\right)\right. \\
& \left.+k_{4}\left(\hat{Q}_{\text {egrl }}-Q_{\text {egrl }}\right)\right)
\end{aligned}
$$

The following conditions for $\lambda_{2}, k_{3}$ and $h_{3}$ :

$$
\lambda_{2}<0, \quad k_{3}>0, \quad h_{3}=-\frac{k_{4}}{k_{3}}
$$

ensures that $\dot{V}_{2}<0$. Conditions (15) and (21) describe the regions of the tuning parameters for which the estimation error converges asymptotically to zero in finite time. The magnitude of $\lambda_{1}$ and $\lambda_{2}$ determines the convergence speed of the observer. It is important to note that the convergence of the observer does not depend on $F_{a v t}, F_{a v c}$, $F_{\text {sural }}$ and $F_{c o l}$, which confirms that the estimation of the LP-EGR mass flow rate can be considered independently from the air mass fraction.

\section{AIR FRACTION ESTIMATION}

In comparison with the approach presented in Wang [2008], the air fraction estimation proposed in this section uses the measurement obtained from the UEGO sensor $\left(F_{a v t}\right)$ as the system output. The advantage is that the complete air fraction dynamics is thus taken into account during the estimation.

Consider the LPV representation of (2)-(5) as follows:

$$
\begin{aligned}
& \dot{X}=A(\rho) X+W(\rho)+\xi_{x} \\
& y=C X+\xi_{y}
\end{aligned}
$$

where $\xi_{x}$ is a stochastic process vector, $\xi_{y}$ is the measurement noise, $X=\left[F_{\text {avt }} F_{\text {avc }} F_{\text {sural }} F_{c o l}\right]^{T}, C=\left[\begin{array}{lll}1 & 0 & 0\end{array}\right]$,

$$
\begin{aligned}
& A(\rho)=\left[\begin{array}{cccc}
-\rho_{1}-\rho_{2} & 0 & 0 & \rho_{1} \\
\rho_{3} & -\rho_{3}-\rho_{4} & 0 & 0 \\
0 & \frac{\rho_{5}}{V_{\text {sural }}} & -\frac{\rho_{5}}{V_{\text {sural }}} & 0 \\
\rho_{6} & 0 & \frac{\rho_{5}}{V_{\text {col }}} & -\frac{\rho_{5}}{V_{\text {col }}}-\frac{\rho_{6}}{V_{\text {col }}}
\end{array}\right] \\
& W(\rho)=\left[\begin{array}{c}
-P C O \rho_{2} \\
\rho_{4} \\
0 \\
0
\end{array}\right]
\end{aligned}
$$

with 
$\rho_{1}=\frac{R T_{a v t}}{p_{a v t} V_{a v t}}\left(Q_{a i r}+Q_{e g r l}+Q_{e g r h}\right), \quad \rho_{2}=\frac{R T_{a v t}}{p_{a v t} V_{a v t}} Q_{f}$,

$\rho_{3}=\frac{R T_{\text {air }}}{p_{\text {air }} V_{\text {avc }}} Q_{e g r l}, \quad \rho_{4}=\frac{R T_{\text {air }}}{p_{\text {air }} V_{\text {avc }}} Q_{\text {air }}$

$\rho_{5}=\frac{R T_{\text {col }}}{p_{c o l}}\left(Q_{a i r}+Q_{e g r l}\right), \quad \rho_{6}=\frac{R T_{\text {col }}}{p_{\text {col }}} Q_{\text {egrh }}$

$\rho$ is a vector that contains all the time-varying parameters. In the general case, the vector consists of $n_{\rho}$ varying parameters $\left[\rho_{1}, \rho_{2}, \ldots, \rho_{n_{\rho}}\right]$ where each varying parameter $\rho_{i}$ is bounded by a minimum and maximum value $\rho_{i}$ and $\overline{\rho_{i}}$. The admissible values of the vector $\rho$ are constrained in an hyperrectangle in the parameter subset $\Theta \subset \mathbb{R}^{n_{\rho}}$ with $N=2^{n_{\rho}}$ vertexes $\left\{v_{1}, v_{2}, \ldots v_{N}\right\}$. The images of the matrix $A(\rho)$ for each vertex $v_{i}$ corresponds to a set $\left\{\Omega_{1}, \ldots, \Omega_{N}\right\}$. The components of the set $\left\{\Omega_{1}, \ldots, \Omega_{N}\right\}$ are the extrema of a convex polytope that contains the images for all admissible values of $\rho$ if the matrix $A(\rho)$ depends linearly on $\rho$. For more details on polytopic models of varying systems (see Angelis [2001]). The bounds $\rho_{i}$ and $\overline{\rho_{i}}$ are established experimentally by calculating the maximum and minimum values of the parameter vector $\rho$ over a representative operating range of the engine. Consider the following LPV Luenberger-like observer:

$$
\dot{\hat{X}}=A(\rho) \hat{X}+W(\rho)+L\left(F_{a v t}-\hat{F}_{a v t}\right)
$$

where $L$ is a constant observer gain vector that ensures the asymptotic stability of the estimation error for all $\rho \in \Theta$. This approach may give relatively conservative results when compared with a $\rho$ dependent observer gain $L(\rho)$. However, its simplicity to implement, its low calculation load and the good results that can be obtained suggest that (25) is an appropriate approach for this application. The observer gain is obtained according to the following theorem.

Theorem 1 (Feron et al. [1992]): Consider system (22). If there exists a symmetric positive definite matrix $P>0$, a matrix $Y$ and a symmetric matrix $X$, for a given diagonal process covariance matrix $V>0$ and a diagonal measurement covariance matrix $W_{y}>0$, such that the following linear matrix inequality is satisfied for all $i \in[1 \ldots N]$ :

$$
\begin{gathered}
A_{i}^{T} P+P A_{i}+C^{T} Y+Y^{T} C+I \prec 0 \\
{\left[\begin{array}{cc}
X & W_{y}^{\frac{1}{2}} Y \\
Y^{T} W_{y}^{\frac{1}{2}} & P
\end{array}\right] \succ 0 \quad L=Y P^{-1}}
\end{gathered}
$$

subject to:

$$
\arg \min _{P, X, Y}\{\operatorname{Tr}(V P)+\operatorname{Tr}(X)\}
$$

then, (25) is an observer of system (22) for all $\varphi \in \Theta$.

Note that Theorem 1 is formulated in terms of LMIs, which can be easily solved numerically using convex optimization methods. In order to ensure that the effect of the estimation of $Q_{\text {egrl }}$ does not destabilize the air fraction observer, the only condition to fulfill is to consider that $\hat{Q}_{\text {egrl }}$ does not drive $\rho$ out of the defined hyperrectangle. As long as this condition is satisfied, both estimators simultaneously converge asymptotically to zero in finite time.

\section{ESTIMATION RESULTS}

In this section, the performance of the estimators combined is evaluated using an engine model that has been previously validated with experimental measurements as a reference. The evaluation is done with the engine model due to the lack of reliable intake manifold air fraction measurements under transient conditions in the actual engine benchmark. The reference model validation has been done using 147 engine operating conditions at steady-state and in transient conditions using the NMVEG cycle as well as with two additional engine cycles. An accuracy better than $10 \%$ (with respect to the benchmark measurements) has been obtained for most of the operating conditions, which allows considering the model to be representative of the engine. The model validation results are not presented in this work due to space limitations.

\subsection{LP-EGR mass flow rate estimator evaluation}

Figure 3 shows the results obtained using the sliding mode estimator presented in Section 4 (a zoom in has been done in order to better illustrate its performance). The estimator parameters used in the simulation are given in Table 1.

Table 1. Sliding mode estimator simulation parameters

\begin{tabular}{|c|c|}
\hline Parameter & Value \\
\hline$k_{1}$ & 0.003 \\
$k_{2}$ & 0.04 \\
$\lambda_{1}$ & -0.005 \\
$k_{3}$ & 0.02 \\
$k_{4}$ & 0.03 \\
$\lambda_{2}$ & -0.03 \\
\hline
\end{tabular}

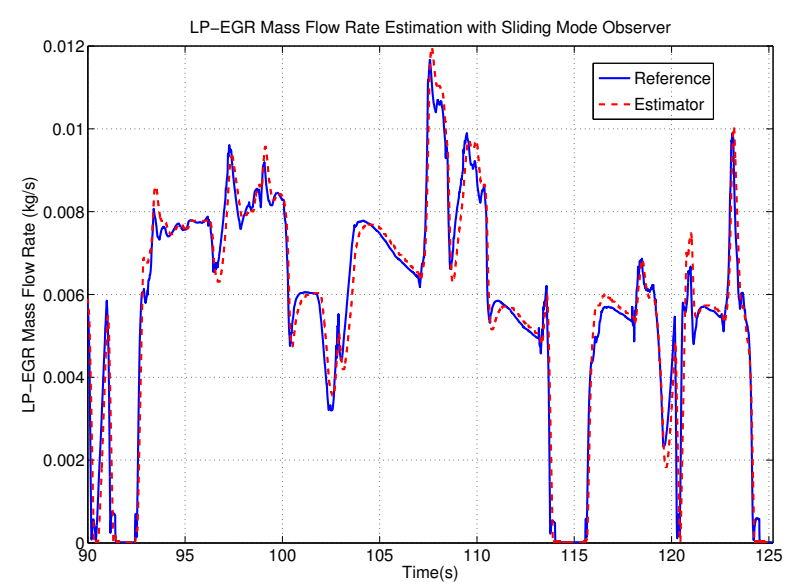

Fig. 3. Zoom on the LP-EGR mass flow rate estimator results

As depicted in Figure 3, the observer quickly converges to the reference LP-EGR mass flow rate, as expected from the results of Section 4. Figure 4 presents the LP-EGR mass flow rate estimation error for a larger time interval 
than the one associated with Figure 3. The observer responds quickly when the LP-EGR valve is suddenly opened or closed. Note that the error remains bounded by $\left|Q_{\text {egrl }}-\hat{Q}_{\text {egrl }}\right|<\left|\lambda_{1}\right|$, which ensures the robustness of the estimation.

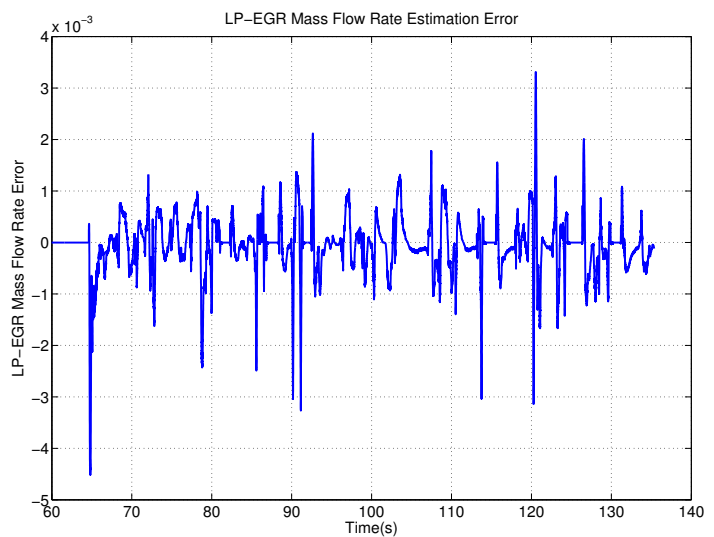

Fig. 4. LP-EGR mass flow rate estimator error

\subsection{Air fraction observer evaluation}

An evaluation of the air mass fraction observer presented in Section 5 is performed using the estimation of the LPEGR mass flow rate obtained in the previous subsection. The bounds of the parameter vector $\rho$ are found using experimental measurements over a representative engine operating conditions. The resulting are given in Table 2.

Table 2. Bounds on the parameter vector
\begin{tabular}{|c|c|c|}
\hline Parameter & ValueMin & ValueMax \\
\hline$\rho_{1}$ & 8.33 & 49.02 \\
$\rho_{2}$ & 0 & 2.17 \\
$\rho_{3}$ & 0 & 2.98 \\
$\rho_{4}$ & 0.98 & 9.38 \\
$\rho_{5}$ & 0.0051 & 0.033 \\
$\rho_{6}$ & 0 & 0.0073 \\
\hline
\end{tabular}

Applying Theorem I with $V=0.01 \times I^{n \times n}$ and $W_{y}=0.01$ (the respective process and measurements noise covariance matrices added in the model), the following observer gain is obtained as:

$$
L=[1.360 .631 .001 .21]^{T}
$$

Figure 5 illustrates the observer effectiveness over the same time interval as the one used in Figure 3. The intake manifold air fraction is estimated as expected from the results of Theorem 1 .

\section{CONCLUSIONS}

In this paper, two 0D model-based observers are proposed to simultaneously estimate the LP-EGR mass flow rate and the air fraction in the intake manifold. The LP-EGR mass flow rate observer was designed using the sliding mode approach while the air fraction observer was obtained using a robust LPV Kalman filter approach. The estimators convergence is guaranteed using the Lyapunov stability and the LMI framework for the LP-EGR mass flow rate and the air fraction, respectively. The effectiveness of the estimators is shown using a model validated on experimental data.

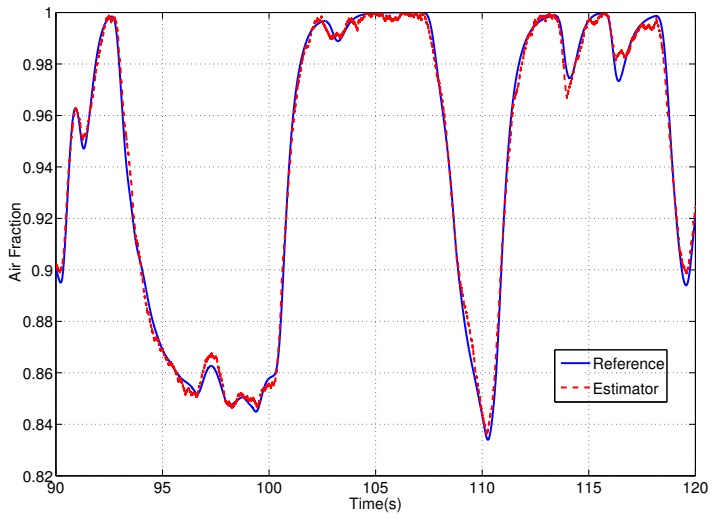

Fig. 5. Zoom of the intake manifold air fraction estimation

\section{REFERENCES}

K. Akihama, Y. Takatory, K. Inagaki, S. Sasaki, and A. Dean. Mechanism of the smokeless rich diesel combustion by reducing temperature. SAE Technical Paper 2001-01-0655, doi:10.4271/2001-01-0655, 2001.

M. Alriksson and I. Denbrantt. Low temperature combustion in a heavy duty diesel engine using high levels of EGR. SAE Technical Paper 2006-01-0075, doi:10.4271/2006-01-0075, 2006.

M. Ammann, N. Fekete, L. Guzella, and A. Glattfelder. Model-based control of the VGT and EGR in a turbocharged common-rail diesel engine: theory and passenger car implementation. SAE World Congress, Detroit, USA, Paper 2003-01-0357, 2003.

G. Angelis. System Analysis, Modelling and Control with Polytopic Linear Models. PhD Thesis. Technische Universiteit Eindhoven, Eindhoven, 2001.

J. Chauvin, G. Corde, and N. Petit. Constrained motion planning for the airpath of a diesel HCCI engine. Proceedings of the 45th IEEE conference on decision and control, pages 3589-3596, 2006.

J. Chauvin, G. Corde, N. Petit, and P. Rouchon. Motion planning for experimental airpath control of a diesel homogeneous charge-compression ignition engine. Control Engineering Practice, 16:1081,1091, 2008.

E. Feron, S. Boyd, and L. El Ghaoui. Numerical methods for $\mathrm{H} 2$ related problems. American Control Conference, pages 2921-2922, 1992.

A. Hribernik. The potential of the high and low-pressure exhaust gas recirculation. Proceeding of the SAE conference, Paper 2002-04-0029, 2002.

I. Kolmanovski, J. Sun, and M. Druzhinina. Charge control for direct injection spark ignition engines with EGR. Proceedings of the 45th IEEE conference on decision and control, 34-38, 2000.

W. Perruquetti and J.P Barbot. Sliding Mode in Engineering. Marcel Dekker, 2002.

T. Ryan and A. Matheaus. Fuel requirements for HCCI engine operation. SAE transactions-Journal of Fuels Lubricants, 112:1143-1152, 2003.

J. Wang. Air fraction estimation for multiple combustion mode diesel engines with dual-loop EGR systems. Control Engine Practice 16, 1479-1468, 16, 2008. 